\title{
Pulse Combustion: The Importance of Characteristic Times
}

\author{
J. O. KELLER and T. T. BRAMLETTE \\ Combustion Research Facility, Sandia National Laboratories, Livermore, CA 94550
}

J. E. DEC

Department of Mechanical Engineering, University of Michigan, Ann Arbor, MI

and

C. K. WESTBROOK

Lawrence Livermore National Laboratory, Livermore, CA

\begin{abstract}
The response of a valved pulse combustor to changes in the relative timing between the resonant pressure wave and the instantaneous energy release rate has been examined. Experiments were designed to examine the pulse combustor's response to independent changes in the experimental conditions that resulted in nearly independent changes in the fluid dynamic species mixing time, the fluid dynamic mixing time of cold reactants with hot products, the characteristic chemical kinetics time, and the characteristic resonance time. The time scales considered in this study were adjusted independently to modify the coupling between the instantaneous energy release rate and the resonant pressure wave, thereby modifying the magnitude of the pressure oscillations and altering the frequency of operation. All of these experimental observations of the pulse combustor response to variations in characteristic time scales are interpreted in terms of Rayleigh's criterion.
\end{abstract}

\section{INTRODUCTION}

Pulse combustion is a very old technology. The phenomenon of combustion-driven oscillations was first observed in 1777 [1], subsequently explained by Lord Rayleigh [2] in 1878, and used in a variety of applications around the turn of the century [3-5]. One of the better known examples of a pulse combustor is the German V-1 "Buzz Bomb" of World War II; an excellent review of this work is given in [6]. In recent years, there have been several comprehensive reviews of the extensive literature associated with the concept [713], as well as three conferences devoted to pulse combustion technology, the proceedings of which document current research and development activity [14-16]. Although the technology of pulse combustion has been known for many years, devices using pulse combustion have not been implemented widely despite their many attractive characteristics. Compared to conventional combustion systems, their heat transfer rates are a factor of two to five higher than normal turbulent values $[17,18]$, their combustion intensities are up to an order of magnitude higher, their emissions of oxides of nitrogen are a factor of three lower, their thermal efficiencies are up to $40 \%$ higher, and they may be self-aspirating, obviating the need for 
a blower. This combination of attributes can result in favorable economic tradeoff with conventional combustors in many applications.

Most of the research on pulse combustors has been directed toward applied examinations of the engineering aspects of pulse combustors: heat transfer, efficiency, frequency of operation, pollutant formation, etc. A review of the current literature on pulse combustion reveals a lack of information on the fundamental fluid dynamic, combustion, and heat transfer processes occurring in pulse combustors. In situ measurements of velocity, temperature, and species concentration are sparse, when available at all. As a result the design and development of pulse combustors has proceeded largely by trial and error, a method that is time-consuming and costly, and that does not guarantee an optimum design. The most severe problem facing the development of pulse combustion systems is the ability to predict a priori how to make these systems resonate [19]. There is confusion as to what factors affect the amplitude of combustor pressure oscillations. Blomquist et al. [20] found that the amplitude of pressure oscillations was proportional to "heat input," while Katsnel'son et al. [21] state that oscillation amplitude is a much stronger function of "excess air" than of "heat release". There is also uncertainty over the behavior of frequency as a function of geometry, energy input, and mass input. Zinn [22] states that the pulse combustor can be modeled as a Helmholtz resonator, while Dec and Keller [23] found that the frequency of operation is a function of the magnitude of the energy input and of the magnitude of the mass flux. These results indicate that a Helmholtz resonator model is insufficient to predict the frequency of operation. These fundamental questions must be answered before the prediction of an optimum resonant condition is possible.

According to Rayleigh's criterion [2], the phase relationship between the resonant pressure wave and the release of energy from chemical reactions will control the operation of the pulse combustor. Therefore, in the present work a systematic study was carried out to understand fully the implications of Rayleigh's criterion on the pulse combustion process and its frequency of operation. The phase relationship between the instantaneous energy release rate and the resonant pressure wave is governed by the different characteristic times of the system (a species mixing time, a fluid dynamic mixing time, a chemical kinetics time, and a resonance time). The total delay time prior to energy release, and hence the phase relationship between the energy release and the resonant pressure wave, is assumed to be a monotonically increasing function of these nearly independent characteristic times. This total time must be some fraction of the characteristic resonance time of the system if Rayleigh's criterion is to be satisfied.

An experimental investigation was performed in which each of the characteristic times contributing to the total ignition delay time was altered independently, while the natural resonance time remained approximately constant. In another series of experiments, the natural resonance time was altered while the total ignition delay time remained constant. In both cases, the response of the combustor was determined by measuring the combustion chamber pressure and its characteristic time of operation. It was found that by suitable normalization, all of the data obtained during this investigation could be collapsed onto a single curve, showing clearly the relationship between the magnitude of the pressure oscillations and the frequency of operation.

\section{BACKGROUND}

\section{Rayleigh's Criterion}

The first recorded statement on the requirements necessary for sustained combustion-driven oscillations was made by Lord Rayleigh in 1878 [2], very elegantly and without proof. Stated briefly, the point of energy release must be in phase with and at the point of maximum pressure to reinforce the resonant pressure wave. Presented in Fig. 1 is a graphic representation of Rayleigh's criterion; a similar discussion is given by Wood [24]. Consider an instantaneous source of energy release, $Q$, which in turn causes an instantaneous pressure rise. If the energy release is at the point of maximum pressure, $3 \pi / 2$, the amplitude of the pressure oscillation is increased with no effect on 


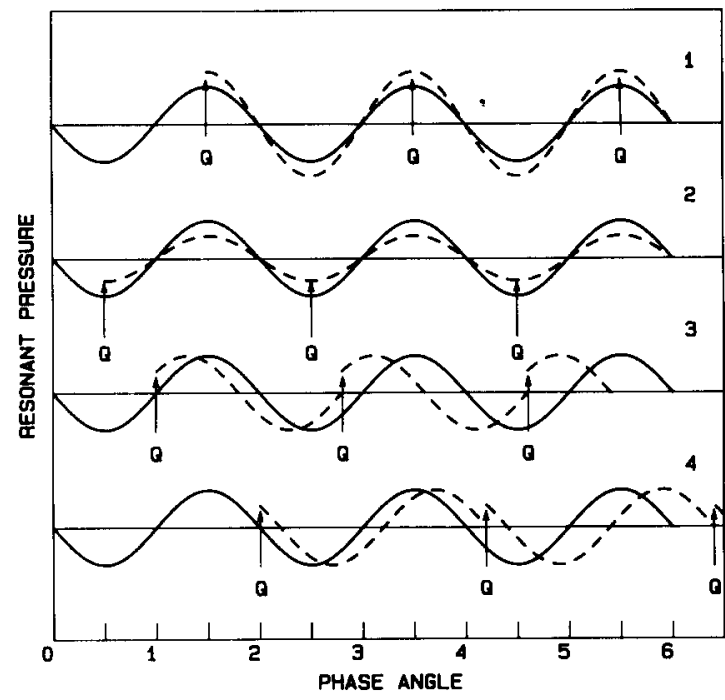

Fig. 1. Schematic representation of Rayleigh's criterion. Here $Q$ represents an instantaneous source of energy release. The solid curve is the undisturbed combustion chamber pressure, and the dashed curve is the pressure after modification by the point source of energy release.

the frequency (case 1 ). If the energy release is at the point of minimum pressure, $\pi / 2$, the amplitude of the pressure oscillation is decreased with no effect on frequency (case 2). If the energy release occurs at $\pi$ the frequency will increase with no effect on the pressure field amplitude (case 3). If the energy release occurs at $2 \pi$ the frequency will decrease with no effect on the pressure field amplitude (case 4). These cases represent extreme conditions where the phase relationship between the energy release and the pressure oscillation affects only the amplitude of the pressure or the frequency of operation. However, if the energy release is at some location other than $n \pi / 2$ then both the amplitude of the pressure wave and the frequency of operation will be affected. It is most common to focus attention primarily on the effect of the phase relationship on the magnitude of the pressure oscillations, but in the present study changes both in the magnitude of the pressure oscillations and the frequency were found to be significant.

Recent work in pulse combustion has alluded to these predictions of Rayleigh's criterion. Keller and Westbrook [25] studied the response of a pulse combustor to changes in the homogeneous chemical kinetic ignition delay time. They found that by decreasing the homogeneous chemical kinetic ignition delay, their Helmholtz-type pulse combustor became "detuned" and operated less stably. This behavior was interpreted as a mismatch in the phase relationship between the instantaneous energy release rate and the resonant pressure wave. Tsujimoto and Machii [26] and Vishwanath and Priem [27] modeled the effects of varying the total ignition delay on the performance of a pulse combustor. In both of these studies it was found that changes in the ignition delay time caused changes in both the pressure oscillation amplitude and the frequency of operation.

The mechanisms that eventually lead to energy release in the pulse combustor involve many complex and coupled processes. The problem is highly elliptic, and the characteristic time scales of the fluid dynamic mixing, the characteristic chemical kinetics time, and the natural resonant time may all be of the same order. Guided by earlier studies, the present work assumes that the controlling mechanism that leads to the release of energy is a result of several subprocesses, each with its own distinct characteristic time. For the Helmholtz combustor used in this study, four characteristic times have been identified:

1. the characteristic time required to mix the fuel with the oxidizer, a species mixing time $\tau_{\text {species }}$;

2 . the characteristic time required to mix the reactants with the hot products, raising the temperature of the reactants to the ignition temperature $\tau_{\text {mixing }}$;

3 . the characteristic time for the chemical reactions to occur $\tau_{\text {kinetic }}$;

4. the characteristic resonance time $\tau_{\text {resonant }}$.

The total ignition delay time $\tau_{\text {total }}$ for the release of energy to occur is assumed to be a function of the first three of these characteristic times:

$$
\tau_{\text {total }}=f\left(\tau_{\text {species }}, \tau_{\text {mixing }}, \tau_{\text {kinetic }}\right) .
$$

It is further assumed that $\tau_{\text {total }}$ is a monotonically increasing function of these characteristic times; for example, it is assumed that if $\tau_{\text {mixing }}$ increases so does $\tau_{\text {total }}$. The phase relationship between the 
release of energy and the resonant pressure wave is assumed to be controlled by the relative magnitude between this total ignition delay time and the resonant pressure wave. This hypothesis was tested by measuring the response of the system to changes in each of these times. Although in this system these times are highly coupled, carefully designed experiments insured that changes in the characteristic time of interest dominated all other effects. These experiments were designed to measure the influence of these characteristic times on the system, not the times themselves.

\section{EXPERIMENTAL FACILITY AND CHARACTERISTIC TIME SCALES}

\section{Experimental Facility}

Figure 2 presents a schematic of the combustor system used in this study. One-way valves (flapper-valves) are located in the reactant supply lines just upstream of the axisymmetric mixing chamber. These valves open and close depending on the pressure difference between the supply lines and the chamber. The combustor was operated with two different types of species mixing $\left(\tau_{\text {species }}\right)$. In the conventional mixing configuration, the reactants entered the mixing chamber separately in a counterflowing fashion. Alternatively, in the second configuration the fuel and air were premixed upstream of the arr flapper-valve and then introduced into the mixing chamber. Dec and Keller [23] have compared the operation of this combustor for both premixed and conventional injection systems and found that this combustor operated favorably in this premixed mode, eliminating many unknowns associated with the conventional injection system. For all but one operating condition presented in this work the system was operated premixed.

The mean mass flow rates of the reactants were controlled and metered by means of sonic nozzles. These flow rates are reported in terms of a mass flux that is calculated by dividing the measured mean mass flow rates by the cross-sectional area of the combustion chamber. The reactants flow from the mixing chamber into a square cross-

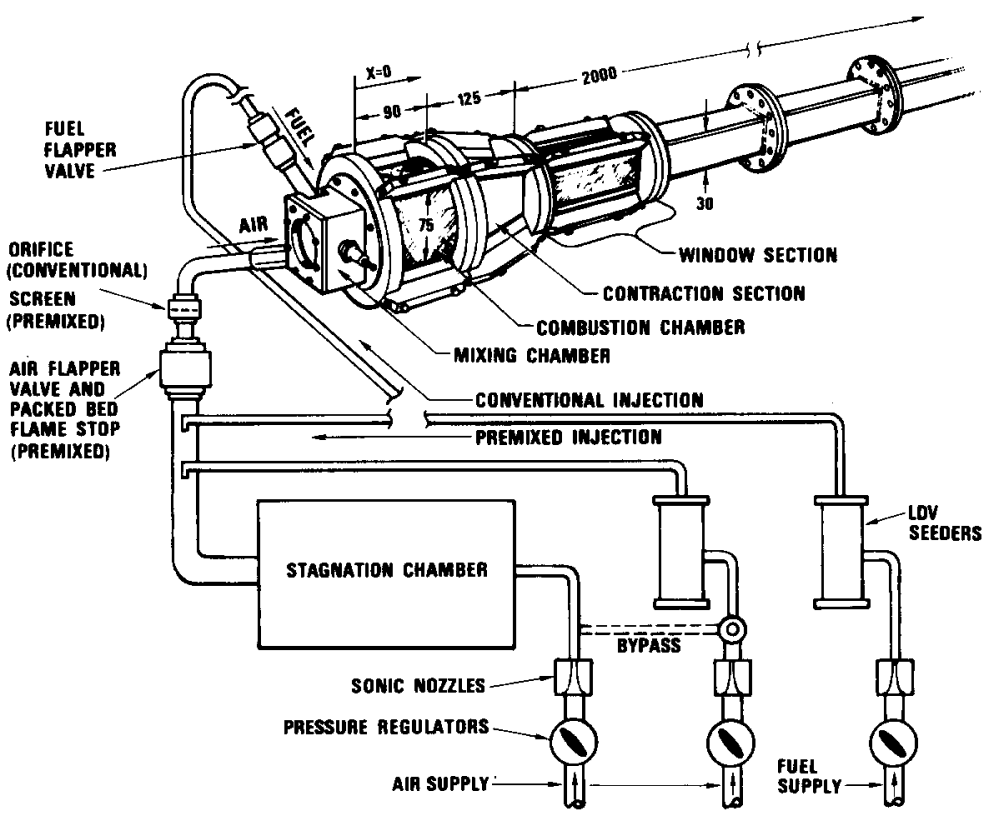

Fig. 2. Schematic of the valved pulse combustor. Shown is the reactant delivery system for both the conventional and the premixed operation. Dimensions are shown in millimeters. 
section combustion chamber, $75 \mathrm{~mm}$ on a side and $90 \mathrm{~mm}$ in length. A square contraction section follows the combustion chamber and joins the combustion chamber to the tailpipe. The tailpipe is made of a variable number of $200 \mathrm{~mm}$ sections, allowing the total length of the tailpipe to be altered, see Fig. 2.

\section{Diagnostics}

Measurements of characteristic cycle time, and combustion chamber pressure were obtained for this study. The data presented here were averaged over many cycles (mean data). The data acquisition rate was maintained at $10 \mathrm{kHz}$, a rate sufficiently fast to accurately resolve the combustor operating frequency of $50-150 \mathrm{~Hz}$. To include low frequency information $(1 \mathrm{~Hz})$, data were taken for periods of at least $10 \mathrm{~s}$, providing 100,000 individual measurements from which the mean stationary statistics were calculated. Both the first (P) and second (Prms) moments of the pressure probability distribution function (PDF) were calculated and are presented. A complete description of the facility can be found in [28].

\section{Chemical Kinetics Time Scales}

Keller and Westbrook [25] and Westbrook and Keller [29] used a detailed chemical kinetics model to estimate the characteristic chemical kinetic time scales in the pulse combustor. The principle features of this model are well documented in [25, 29], however, a brief discussion of this model is repeated here for convenient reference.

The model follows the time evolution of a given sample of gas that consists initially of a mixture of fuel, air, and residual products from previous cycles. The computations use the HCT code [30] with a detailed reaction mechanism that has been thoroughly tested for the fuel and conditions of this study [31-33]. The pressure during ignition is assumed to be constant at $101.3 \mathrm{kPa}$, spatial variations in temperature and species concentrations are neglected, and energy losses during ignition are not considered. These assumptions are reasonable as long as the homogeneous chemical ignition delay time is much shorter than the fluid dynamic mixing time, which is much shorter than the overall period of the pulse combustor $\left(\tau_{\text {kinetic }} \&\right.$ $\tau_{\text {mixing }} \ll \tau_{\text {resonant }}$ ).

Keller and Westbrook were guided by this model in their study of the influence of fuel composition on the behavior of pulse combustion [25]. Based on their previous work Westbrook and Keller applied this model to the calculation of the homogeneous chemical ignition delay time in the search for a set of reactants which would lengthen the characteristic chemical kinetics time [29]. They found that the addition of small amounts of $\mathrm{N}_{2}$ or $\mathrm{CO}_{2}$ would significantly lengthen $\tau_{\text {kinetic }}$. As shown below, the use of $\mathrm{N}_{2}$ as a diluent will not significantly affect the physical or thermal properties of the reactants, and hence only $\tau_{\text {kinetic }}$ will be affected. Thus, $\mathrm{N}_{2}$ was chosen as the diluent to control $\tau_{\text {kinetic }}$.

The physical and thermal properties are invariant to the addition of small amounts of $\mathrm{N}_{2}$ as a diluent for several reasons. Both $\mathrm{O}_{2}$ and $\mathrm{N}_{2}$, being diatomic molecules with similar molecular weights, have similar physical and thermal properties. Moreover, $\mathbf{N}_{2}$ is already the major constituent of air, so small changes in the amount of $\mathrm{N}_{2}$ will cause negligible changes in the physical and thermal properties of the reactants. Consequently the adiabatic flame temperature (for lean combustion) and the fluid dynamic behavior are invariant whether $\mathrm{O}_{2}$ or $\mathrm{N}_{2}$ is chosen as the diluent.

Although the thermal and physical properties are invariant to the species chosen as the diluent $\tau_{\text {kinetic }}$ is not species independent. Consider a simple one-step reaction rate of the Arrhenius form with $\mathrm{CH}_{4}$ as the fuel

\section{Reaction Rate $=$ Const $\left[\mathrm{O}_{2}\right]\left[\mathrm{CH}_{4}\right] \exp (-\mathrm{E} / \mathrm{RT})$.}

Because the adiabatic flame temperature is not a function of the species used as a diluent $\left(\mathrm{O}_{2}\right.$ or $\mathrm{N}_{2}$ ), the exponential term is also species independent. However, the preexponential term is a function of $\mathrm{O}_{2}$ concentration, and hence is species dependent. Thus, as the $\mathrm{N}_{2}$ concentration increases (displacing $\mathrm{O}_{2}$ ) $\tau_{\text {kinetic }}$ increases through a decrease in the probability of a collision occurring between $\mathrm{O}_{2}$ and $\mathrm{CH}_{4}$. Hence, only $\tau_{\text {kinetic }}$ (and therefore $\tau_{\text {total }}$ ) will be altered. 


\section{Fluid Dynamic Time Scales}

The mixing model of Rife and Heywood [34, 35] was recently extended and applied to the injection process that occurs in a pulse combustor [36]. This model is based upon the assumption of similarity profiles obtained in nonreacting turbulent jets, and thus it incorporates all of the physical transport processes (turbulent and molecular mixing) that are responsible for these "universal profiles." Bramlette [36] hypothesized that for the case of "fast" kinetics (i.e., when the chemical ignition delay time scale is much less than the acoustic and fluid dynamic time scales of the combustor, $\tau_{\text {kinetic }}$ $\left.\ll \tau_{\text {mixing }}\right)$, the rate of fluid dynamic mixing may be used to predict the timing and instantaneous rate of energy release in a pulse combustor.

Bramlette performed a series of calculations to determine the sensitivity of the predicted mixing rate to mass flow rate, jet radius, combustion product temperature, density ratio, and the temperature required for chemical ignition to begin, $T_{\operatorname{mix}}$ [36]. The mass flow rate was found to have the most significant effect. Bramlette showed that the mixing time $\tau_{\text {mixing }}$ scaled with $r_{0} / u_{0}$, where $r_{0}$ is the jet radius and $u_{0}$ is the inlet jet velocity. In the present work, $\tau_{\text {mixing }}$ was modified by changing the mass injection rate and, hence, the injection velocity.

\section{EXPERIMENTAL INVESTIGATION}

\section{Effects of Characteristic Kinetics Delay Time}

Keller and Westbrook [25] established the use of Rayleigh's efficiency $\eta$ as a measure of the behavior of the pulse combustor, where $\eta$ is defined by

$$
\begin{aligned}
& \eta=\text { Const } \int_{0}^{1} \dot{Q}^{\prime}(\zeta) P^{\prime}(\zeta) d \zeta \\
& \text { Const }=\frac{R}{M C_{\mathrm{p}} P \dot{Q}_{\mathrm{t}}}
\end{aligned}
$$

Here, $C_{\mathrm{p}}$ is the specific heat at constant pressure; $R$ is the universal gas constant; $M$ is the mole averaged molecular weight; $P$ is the mean pressure; $P^{\prime}(\zeta)$ is the perturbation of pressure about the mean, $P^{\prime}(\zeta)=P-P(\zeta) ; \dot{Q}$ is the mean energy release rate; $\dot{Q}_{t}$ is the total energy release rate; $\dot{Q}^{\prime}(\zeta)$ is the perturbation of the instantaneous energy release rate about the mean; $\dot{Q}^{\prime}(\zeta)=\dot{Q}$ - $\dot{Q}(\zeta)$; and $\zeta$ represents time normalized by the total cycle time. Shown in Fig. 3 is a comparison of Rayleigh's efficiency $\eta$ and Prms for the conventional injection mode. Figure 3 shows that Prms and $\eta$ exhibit the same trends, suggesting that Prms can be used as a measure of the response of the pulse combustor to changes in the relative

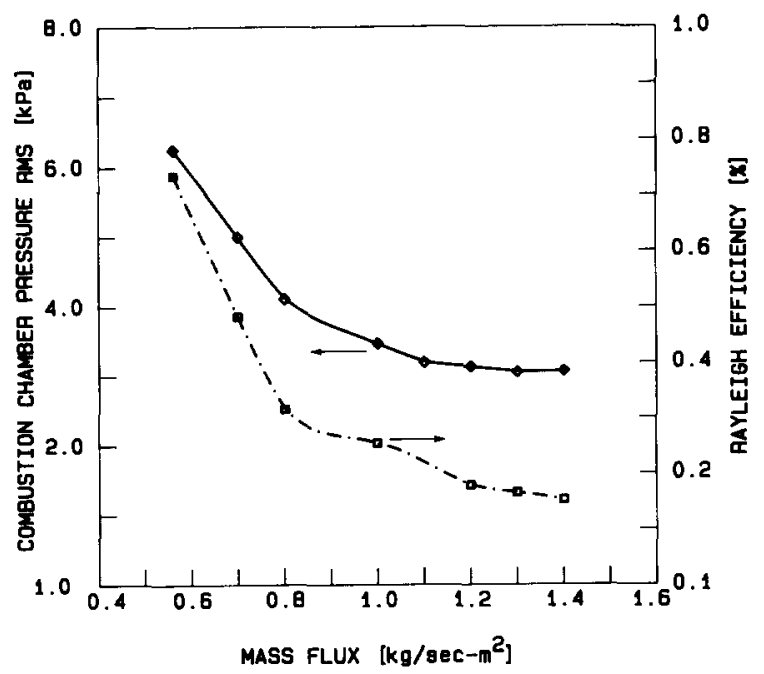

Fig. 3. Comparison of Rayleigh's efficiency (Eq. 3) and the magnitude of the oscillating component of the combustion chamber pressure as a function of mass flux. 
phase between the instantaneous energy release and the resonant pressure wave. For this study Prms is used to measure the response of the pulse combustor to changes in this relative phase relationship.

The effect of systematic dilution of the fuel-air mixture with $\mathrm{N}_{2}$ was studied in a series of experiments in the premixed configuration, with the observed values of the magnitude of the pressure oscillations and frequency plotted in Fig. 4. For each value of the equivalence ratio $\phi$ between 0.6 and 1.2 , the pulse combustor was operated with varying amounts of $\mathrm{N}_{2}$ diluent. Since dilution by an inert species displaces some fuel and air in the incoming reactant mixture, this dilution is actually reflected in Fig. 4 as a net decrease in the fuel mass flow rate. The dashed curves in Fig. 4 show the results when no diluent was added. In every case, dilution by additional $\mathrm{N}_{2}$ resulted at first in an increase in the magnitude of the pressure oscillations until a maximum value was attained (Prms ${ }_{\max }$ ), following which the magnitude of the pressure oscillations steadily decreased. Moreover, throughout the entire sequence of increased dilution, the frequency was observed to decrease monotonically.

Dilution of a given fuel-air mixture reduces the amount of energy released per unit mass of reactant mixture. However, it is clear from the results in Fig. 4 that there is a distinct regime where, in spite of the reduced rate of energy release, dilution enhances the coupling between the instantaneous energy release rate and the pressure oscillations in the combustor. On the basis of Rayleigh's criterion, the observed behavior indicates that without dilution the instantaneous energy release rate is taking place prior to the attainment of the maximum value of the pressure oscillations. Dilution retards the energy release phase and increases the value of $\tau_{\text {kinetic }}$, which increases the value of $\tau_{\text {total }}$, thereby improving the coincidence between the instantaneous energy release rate and the pressure oscillations. As the value of $\tau_{\text {kinetic }}$ is increased further, eventually the instantaneous energy release rate occurs after the time of maximum pressure. Again reasoning on the basis of Rayleigh's criterion, the steady reduction in the correlation between the instantaneous
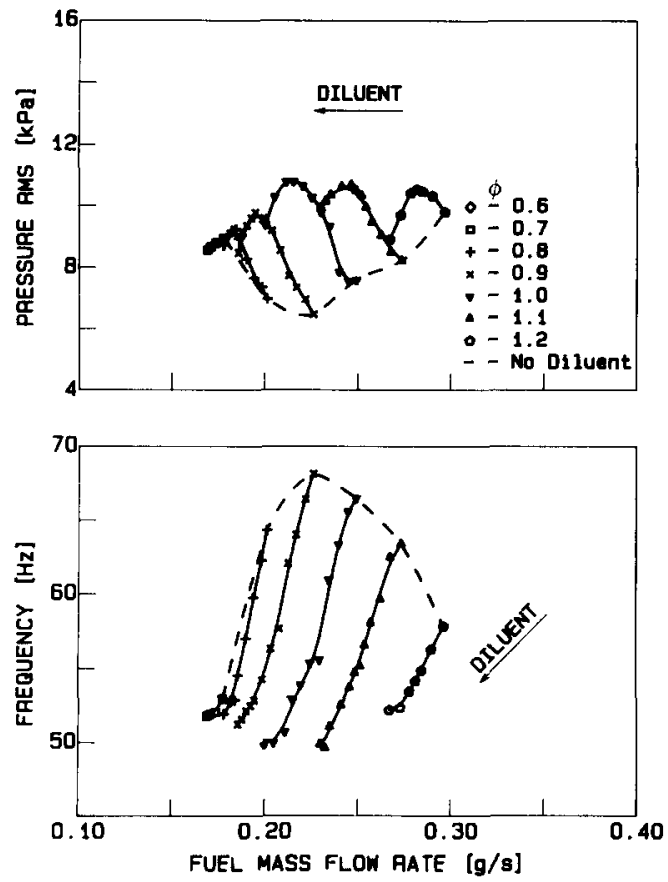

Fig. 4. Combustion chamber pressure rms (upper) and operating frequency (lower) shown as functions of mean fuel-mass flow rate. These data are plotted as lines of constant equivalence ratio. These data were obtained by maintaining the mass flux and the equivalence ratio constant while varying the amount of $\mathrm{N}_{2}$ diluent. Shown are data for equivalence ratios in the range of 0.6 to 1.2 at a mass flux of $1.0 \mathrm{~kg} \mathrm{sec}^{-1} \mathrm{~m}^{-2}$.

energy release rate and the pressure oscillations produces a decrease in the peak values of the pressure oscillations. The monotonic decrease in the operating frequency with $\mathrm{N}_{2}$ dilution observed in Fig. 4 is also predicted from Rayleigh's criterion, as discussed earlier.

The same reasoning has been used previously by Keller and Westbrook [25] to interpret experimental results in which the fuel composition was varied. Using the conventional pulse-combustor configuration, it was observed that when the fuel consisted of methane, the instantaneous energy release rate occurred at a time slightly earlier than the time of peak pressure. When a mixture of methane and ethane was used $\left(0.85 \mathrm{CH}_{4}+\right.$ $0.15 \mathrm{C}_{2} \mathrm{H}_{6}$ ), the process of ignition was accelerated, reducing $\tau_{\text {kinetic }}$. Because the instantaneous energy release rate was already too early (i.e., before the time of peak pressure), accelerating the 
ignition phase further reduced the correlation between the instantaneous energy release rate and the pressure oscillations and reduced the value of Prms.

\section{Effects of Total Energy Release Rate}

As found by other researchers, there is an effect of the absolute magnitude of the energy release rate on the magnitude of the pressure oscillations. According to Rayleigh's criterion (Fig. 1), if the phase relationship between the energy release rate and the pressure oscillations is at a point to create a maximum in Prms (Prms ${ }_{\max }$ ) the influence of the energy release on the frequency is minimized. The frequency at which Prms $_{\max }$ is located is the natural resonant frequency of the device, for the given operating conditions, and geometry. The dependence on the phase relationship between the resonant pressure wave and the energy release rate is eliminated in Prms max $_{\max }$. For a given geometry Prms $_{\max }$ is only a function of the absolute magnitude of the energy release rate.

To illustrate the pressure oscillation's dependence on the magnitude of the energy release rate, $\mathrm{Prms}_{\max }$ for each case in Fig. 4, along with two other mass flux cases, is plotted in Fig. 5. The magnitude of the pressure oscillations (along lines of constant mass flux) increases with increasing equivalence ratio as shown in the data. This increase in $\mathrm{Prms}_{\max }$ continues up to an equivalence ratio of 1.0 , corresponding to increasing energy release rates. Similarly as the equivalence ratio continues to increase into the rich region, $\operatorname{Prms}_{\max }$ decreases slightly, due to lower energy release rate. Furthermore, except at the lowest value of the equivalence ratio, $\mathrm{Prms}_{\max }$ increases with increasing mass flux, since the higher total mass flux is accompanied by a higher fuel mass flux and a correspondingly larger energy release rate.

Two of the curves for constant mass flux are remarkably similar in shape (mass fluxes of $0.8 \mathrm{~kg}$ $\mathrm{s}^{-1} \mathrm{~m}^{-2}$ and $1.0 \mathrm{~kg} \mathrm{~s}^{-1} \mathrm{~m}^{-2}$ ). In contrast, for the highest mass flux of $1.2 \mathrm{~kg} \mathrm{~s}^{-1} \mathrm{~m}^{-2}, \mathrm{Prms}_{\max }$ falls more rapidly with decreasing equivalence ratio than for the other cases. This somewhat anomalous behavior can be explained by the results of the mixing and kinetic models. According to the

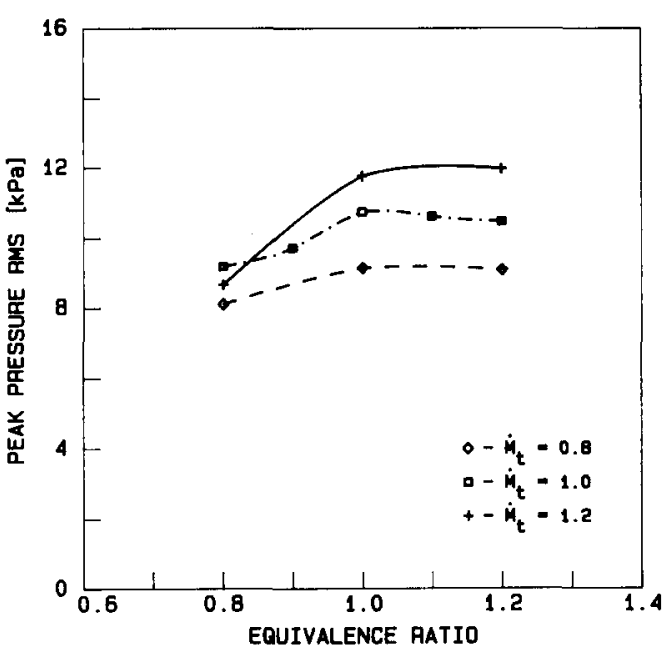

Fig. 5. Prms max $_{\max }$ plotted as a function of equivalence ratio for the three mass flux cases studied $\left(0.8,1.0,1.2 \mathrm{~kg} \mathrm{sec}^{-1} \mathrm{~m}^{-2}\right)$. Prms $_{\max }$ is defined to be the peak value of Prms for each of the constant equivalence ratio cases shown in Fig. 5.

mixing model, $\tau_{\text {mixing }}$ decreases with increasing total mass flux. Therefore, in order to increase $\tau_{\text {total }}$ to achieve a maximum in Prms, $\tau_{\text {kinetic }}$ must be increased a great deal through the addition of a significant quantity of excess $\mathrm{N}_{2}$. Large amounts of dilution can cause a significant change in the temporal shape of the instantaneous energy release rate with time, and this trend may be responsible for the results shown in Fig. 5 at high values of the total mass flux and low values of the equivalence ratio. The effect of the temporal shape of the instantaneous energy release rate on the resonant behavior of the pulse combustor is not well understood, and is an area of current research.

The characteristic chemical kinetics time ( $\left.\tau_{\text {kinetic }}\right)$ was changed by the addition of $N_{2}$, providing control over the total ignition delay $\left(\tau_{\text {total }}\right)$. Under conditions of constant equivalence ratio the amount of fuel in the system is an inverse function of the amount of $\mathrm{N}_{2}$ added, which changes the magnitude of the energy release rate. To remove any effects caused by a change in the energy release rate, additional data were taken at constant fuel flow rate, and thus with a constant average energy release rate (constant adiabatic flame temperature). Figure 6 shows the results of this measurement. The data presented here were obtained by maintaining the mass flow rate of fuel 
and the total mass flux constant and varying the amount of $\mathrm{N}_{2}$ diluent to lengthen the characteristic chemical kinetics time. In the absence of excess diluent, the instantaneous energy release rate occurs prior to the time of peak pressure; as $\mathrm{O}_{2}$ is displaced by excess $\mathrm{N}_{2}$ and the equivalence ratio increases, $\tau_{\text {kinetic }}$ grows larger and $\tau_{\text {total }}$ therefore provides an improvement in the phase relationship between the instantaneous energy release rate and the resonant pressure wave. This increases the magnitude of the pressure oscillations and decreases the frequency. This particular data set did not have sufficient dynamic range to locate Prms $_{\max }$ while keeping the equivalence ratio less than 1.0. Nevertheless, the results shown in Fig. 6 provide a further example of the applicability of Rayleigh's criterion.

\section{Effects of Species Mixing Time}

According to Rayleigh's criterion (Fig. 1), if the energy release rate is before Prms ${ }_{\text {max }}$ and $\tau_{\text {total }}$ is


Fig. 6. Prms (upper) and frequency (lower) shown as a function of equivalence ratio for a constant mass flow rate of fuel $\left(0.22 \mathrm{~g} \mathrm{~s}^{-1}\right)$. These data were obtained by maintaining a constant total mass flux and the mass-flow rate of fuel, and varying the amount of $\mathrm{N}_{2}$ diluent, which causes the equivalence ratio to vary. increased then Prms increases and the frequency will decrease. All the data presented so far were obtained while operating the combustor under premixed conditions, eliminating $\tau_{\text {species }}$. Operating the combustor under a conventional mixing configuration will increase the total mixing time due to a nonzero contribution of $\tau_{\text {species. }}$. When the pulse combustor was operated in the conventional mode $\left(\tau_{\text {species }}>0\right)$, the value of Prms was observed to increase from $6.2 \mathrm{kPa}$ to $7.0 \mathrm{kPa}$, and the frequency was found to decrease from $55 \mathrm{~Hz}$ to $48 \mathrm{~Hz}$.

In all three cases, the dilution of the fuel-air mixture by the addition of $\mathrm{N}_{2}$, the addition of ethane to the methane fuel, and the change from conventional to premixed operation, the observed behavior is easily interpreted in terms of Rayleigh's criterion and the correlation between the time of energy release and the time of maximum pressure in the combustion chamber.

\section{Effects of Natural Resonant Frequency}

Rayleigh's criterion states that it is the relative phase relationship between the instantaneous energy release rate and the resonant pressure wave that is important. As discussed above the natural resonant frequency of this device for a given operating condition is at the point of $\mathrm{Prms}_{\max }$. The natural resonant frequency is a function of both geometry and temperature of the gases in the combustor chamber and the tailpipe. Figures 4 and 6 present data where the total ignition delay time was varied, while the geometry remained constant. Holding the geometry constant maintained the natural resonance time (the inverse of the natural resonance frequency) constant, within the ability to maintain constant temperature. The phase relationship between the instantaneous energy release rate and the resonant pressure wave can also be altered by maintaining the total ignition delay time constant and changing the geometry and, hence, the natural resonance time. This was accomplished by systematically changing the tailpipe length; the results are summarized in Fig. 7, in which Prms and frequency are plotted as functions of tailpipe length. The initial conditions for the baseline case were chosen so that the 

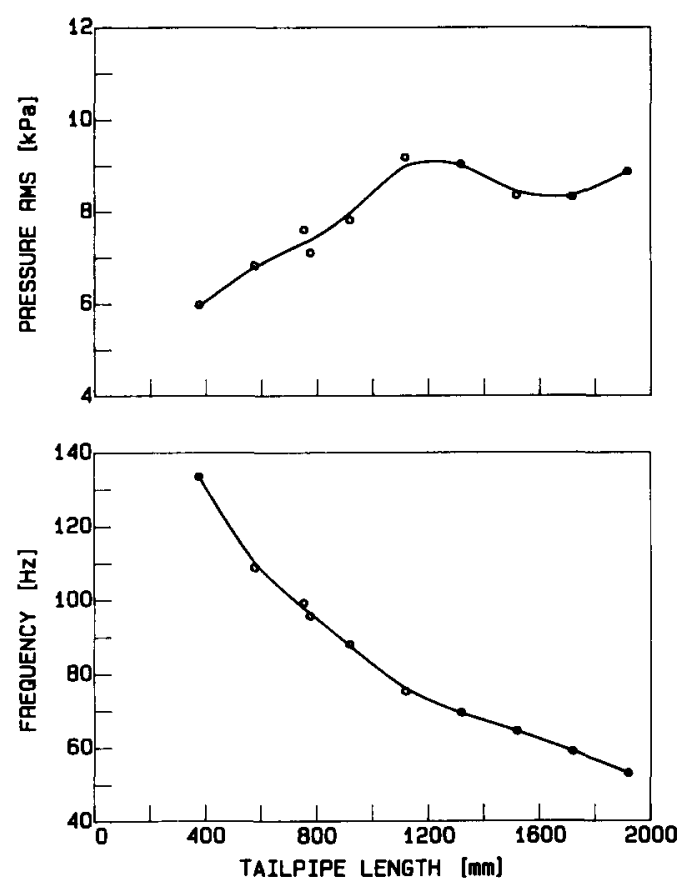

Fig. 7. Prms (upper) and frequency (lower) as a function of the tail pipe length.

instantaneous energy release rate was slightly later than the peak resonant condition. Therefore, when the natural resonance time is lengthened, Prms goes through a maximum and the frequency continues to decrease. The location of this local maximum in Prms is at the position of maximum pressure oscillation reinforcement. This local maximum in Prms is again interpreted in terms of Rayleigh's criterion and the correlation between the resonant pressure wave and the time of energy release. As the tailpipe length continues to increase the magnitude in the pressure oscillation increases slightly. This slight increase may be caused by a number of effects other than the phase matching of the resonant pressure wave with the timing of the energy release, which resulted in the local maximum. The reason for this increase is an area of current investigation.

\section{Pressure Amplitude Versus Frequency}

Rayleigh's criterion predicts that there should be a relationship between the resonant pressure wave and the frequency of operation. The data presented

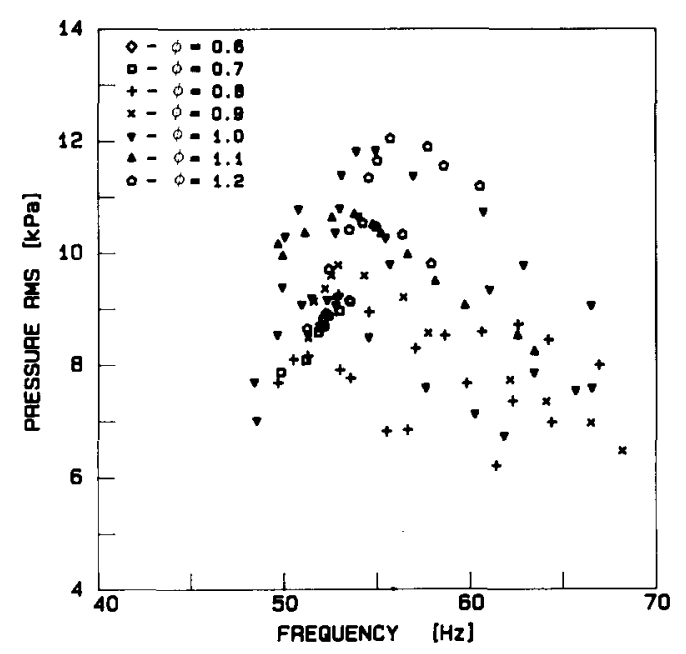

Fig. 8. Prms versus frequency for the data presented in Fig. 5. Also plotted are data taken at mass fluxes of 0.8 and $1.2 \mathrm{~kg}$ $\sec ^{-1} \mathrm{~m}^{-2}$.

in Figures 4-7 were obtained by carefully modifying the phase relationship between the instantaneous energy release rate and the resonant pressure wave. To examine the relationship between the resonant pressure wave and the frequency of operation, the pressure and frequency data presented above are plotted in Fig. 8, showing the pressure rms as a function of operating frequency. The data from Fig. 6 were not included because they did not have sufficient dynamic range to allow determination of the optimum phase relationship between the instantaneous energy release rate and the resonant pressure wave. The data in Fig. 8 show significant scatter, indicating no clear correlation between the two variables.

Many experimental quantities were changing simultaneously, making it difficult to extract information about specific controlling parameters. For example, the natural resonance frequency of the combustor is affected both by the instantaneous energy release rate and by the rate of heat transfer in the tailpipe. To facilitate more meaningful analysis of the above data, the pressure and frequency data were transformed in the following way. The pressure oscillations Prms were normalized by the peak in the pressure oscillation $\left(\mathrm{Prms}_{\max }\right)$, removing the influence of the magnitude of the energy release rate on Prms. The influences of temperature and geometry were 
eliminated by normalizing the frequency by the natural resonant frequency of the device. This natural resonance frequency was determined experimentally as the frequency at Prms ${ }_{\max }$, where, by Rayleigh's criterion, there is no influence on the frequency due to the location of the instantaneous energy release rate. It was assumed that the rate of heat transfer in the tailpipe was unaffected by the addition of diluent. (This assumption is reasonable because the heat transfer was limited by the natural convection on the outside walls). The temperature of the combustion products can be a function of $\mathrm{N}_{2}$ diluent and can produce a change in the effective natural resonance frequency. This effect is weak, because the frequency is a function of the square root of temperature. The influence of temperature on the natural resonance frequency was, however, included by assuming that the change in the natural resonant frequency is a function only of the temperature of the hot gas products, as given by

$f_{\mathrm{n}}=f_{\max }\left(\frac{T_{\mathrm{n}}}{T_{\max }}\right)^{\frac{1}{2}}$.

Here $f_{\mathrm{n}}$ represents the corrected natural resonant frequency; $f_{\max }$ is the frequency at Prms $s_{\max }$ for a given equivalence ratio, mass flux, and geometry; $T_{\mathrm{n}}$ is the adiabatic flame temperature at the actual equivalence ratio and diluent loading; and $T_{\max }$ is the adiabatic flame temperature at peak pressure. Equation 3 therefore represents a correction to changes in the natural resonance frequency caused by changes in the adiabatic flame temperature due to modification in the amount of $\mathbf{N}_{2}$ diluent. Normalizing the frequencies in Fig. 8 by the natural resonant frequency given by $\mathrm{Eq} .3$ and normalizing the pressures by $\mathrm{Prms}_{\max }$ all of the data collapse onto the curve shown in Fig. 9. The only data that appear to be noise in Fig. 9 are those cases from Fig. 7, shown here as circles, which are the extremes in tailpipe length. These data were on the limits of stable operation and, hence, one would expect these data to be noisy. However, all of the other tailpipe lengths fit the normalized data very well. This shows the relationship between the normalized frequency and the magnitude of the pressure oscillations is a function of only the phase relationship between the instantaneous energy release rate and the resonant pressure wave.

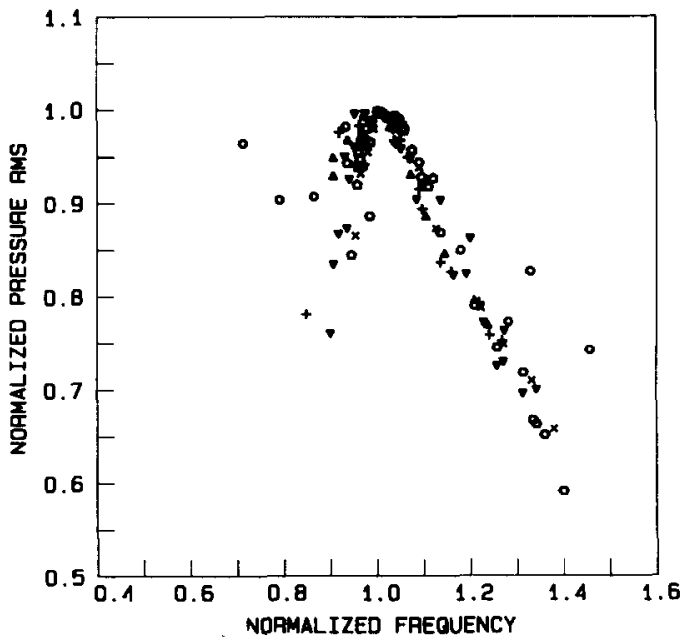

Fig. 9. Normalized Prms versus normalized frequency, showing the relationship between the magnitude of the pressure oscillations and the frequency of operation. Also shown are the data from Fig. 7, which are plotted as circles.

When normalized in this manner the agreement among these data is excellent.

\section{SUMMARY AND CONCLUSIONS}

A systematic study has been performed in which the influence of individual characteristic times on the performance of a Helmholtz-type pulse combustor was examined. It was found that under the conditions examined, the response of this pulse combustor was explained by the phase relationship between the resonant pressure wave and the instantaneous energy release rate. In addition, under the conditions examined, the magnitude of the pressure oscillations and the frequency of operation were stronger functions of this phase relationship than they were of the magnitude of the energy release rate. In fact, in many cases the magnitude of the pressure oscillation increased as the magnitude of the energy release rate $d e$ creased. It has been shown that the relative phase relationship between the temporal location of the instantaneous energy release and the resonant pressure wave can be determined by examination of Prms to systematic changes in $\tau_{\text {kinetic }}$.

The delay time of the instantaneous energy release rate (total ignition delay time) is controlled by four individual processes. These individual processes were modified separately to alter the 
phase relationship between the energy release rate and the resonant pressure wave. It is only through their contribution to the total delay time that the phase relationship between the resonant pressure wave and the instantaneous energy release rate was affected, and it is this total delay time that is the important factor in determining the response of the pulse combustor.

The authors wish to express their gratitude to Ichiro Hongo of Toshiba Corporation for assistance in the laboratory during the long hours of data acquisition. This work was performed at the Combustion Research Facility at Sandia National Laboratories, supported by the U.S. Department of Energy, Office of Energy Utilization Research, Division of Energy Conversion and Utilization Technologies, and at Lawrence Livermore National Laboratory, supported by the U.S. Department of Energy and the Gas Research Institute.

\section{REFERENCES}

1. Tyndall, J., Sound. D. Appelton and Company, New York, 1897.

2. Lord Rayleigh Nature 18:319 (1878).

3. Foa, J. V., Elements of Flight Propulsion. John Wiley and Sons, New York, 1927.

4. Stodola, A., Steam and Gas Turbine. McGraw-Hill, New York, 1927, Vol. 2.

5. Reynst, F. H., in Pulsating Combustion-The Collected Works of F. H. Reynst (N. W. Thring, Ed.), Pergamon Press, New York, 1961.

6. Oppenheim, A. K., British Intelligence objectives subcommittee report no. 1777, Technical Information and Documents Unit, London, England, 1949.

7. Zinn, B. T., Mech. Eng. 36-41 (1985).

8. Zinn, B. T., ASME paper 84-WA/NCA-19, ASME winter annual meeting, New Orleans, December 1984.

9. Putnam, A. A., in Proceedings of the first International Symposium of Pulsating Combustion (D. J. Brown, Ed.), Sheffield, England, 1971, pp. 1-1-1-45.

10. Putnam, A. A., ASME paper 84-WA/NCA-20, ASME winter annual meeting, New Orleans, 1984.

11. Severyanin, V. S., in Proceedings of the First International Symposium of Pulsating Combustion (D. J. Brown, Ed.), Sheffield, England, pp. 13-1-13-20.

12. Severyanin, V. S., in Proceedings of the Symposium on Pulse Combustion Applications, GRI-82/0009.2, Atlanta, GA, 1982, Vol. 1.

13. Putnam, A. A., Belles, F. E., and Kentfield, J. A. C., Prog. Energy Combus. Sci. 12:1 (1986).

14. Proceedings of the First International Symposium of
Pulsating Combustion (Brown, D. J., Ed.), Sheffield, England, 1971.

15. Proceedings of the Symposium on Pulse Combustion Technology for Heating Applications, Argonne National Laboratories report ANL/EES-TM-87, 1980.

16. Proceedings of the Symposium on Pulse Combustion Applications, GRI-82/0009.2, Atlanta, 1982, Vol. 1.

17. Dec, John E. and Keller, Jay O., "Pulse Combustor Tailpipe Heat-Transfer Dependence on Frequency, Amplitude, and Mean Flow Rate," submitted Combust. Flame.

18. Hanby, V. I., J. Eng. Power 91:48-52 (1969).

19. Kardos, P., private communication, Forbes Energy Engineering, Inc., Springfield, 1985.

20. Blomquist, C. A., Clinch, J. M., and Chiu, H. H., in Proceedings of the Symposium on Pulse Combustion Applications, Atlanta, 1982, Vol. 1.

21. Katsnel'son, B. D., Marone, I. Ya., and Tarakanovskii, A. A., Soviet Power Industry Res. Technol. Thermal Eng. Vol. 4-8 (1969).

22. Weinberg, F. J., Advanced Combustion Methods. Academic Press, 1986, p. 151.

23. Dec, J. E., and Keller, J. O., in Proceedings of the 1986 International Gas Research Conference, Toronto, Canada, 1986.

24. Wood, A., Acoustics. Dover, New York, 1966.

25. Keller, J. O., and Westbrook, C. K., in Proceedings of the Twenty-First Symposium (International) on Combustion, The Combustion Institute, Pittsburgh, 1986.

26. Tsujimoto, Y., and Machii, N., Burner, Twenty-First Symposium (International) on Combustion.

27. Vishwanath, P. S., and Priem, R. J., presented as a poster paper at the Twenty-First Symposium (International) on Combustion, Technical University of Munich, West Germany, 1986.

28. Keller, J. O., and Saito, K., Combust. Sci. Technol., 53:137 (1987).

29. Westbrook, C. K., and Keller, J. O., in Proceedings of the 1986 International Gas Research Conference, Toronto, Canada, 1986.

30. Lund, C. M., Lawrence Livermore National Laboratory report UCRL-52504, University of California, Livermore, 1978.

31. Westbrook, C. K., Combust. Sci. Technol. 20:5 (1979).

32. Westbrook, C. K., and Pitz, W. J., Combust. Sci. Technol. 37:117 (1984).

33. Pitz, W. J., Westbrook, C. K., Proscia, W. M., and Dryer, F. L., in Proceedings of the Twentieth Symposium (International) on Combustion, The Combustion Institute, Pittsburgh, 1985, p. 831.

34. Rife, J. M., PhD thesis, Massachusetts Institute of Technology, 1974.

35. Rife, J. M., and Heywood, J. B., SAE Transactions 83:2942-2961 (1974).

36. Bramlette, T. T., Sandia National Laboratory report Sand87-8622, submitted to Combust. Sci. Technol.

Received 1 June 1987; revised 3 March 1988 